\title{
Modeling of Priority Queueing Service in Discrete Event Systems Using Hybrid Petri Nets
}

\author{
Ming-Hung Lin and Li-Chen Fu
}

\author{
Dept. of Computer Science and Information Engineering, \\ National Taiwan University, \\ Taipei, Taiwan, R.O.C \\ E-mail: lichen@ccms.ntu.edu.tw
}

\begin{abstract}
In this paper, we proposed a Hybrid Petri Net (HPN) that extends the framework of the original colored generalized stochastic Petri Net by hiding queues in special places. The Hybrid Petri Net contains ordinary tokens and control tokens. Both of ordinary tokens and control tokens can be exogenous, or can be obtained by a Markovian movement of a token from one place to another place after firing of transition. A control token forces an ordinary token to move instantaneously to another place according to a Markovian routing rule. We show that this new class of Petri Net has product form stationary solution, and establish the non-linear token flow equations that govern it. The stability of Hybrid Petri Net is discussed in this new context.
\end{abstract}

\section{Introduction}

The queueing services represent an example of a much broader class of interesting dynamic systems, which, refer to as "systems of flow." It acts as a very important role in a discrete event system. The use of queueing networks as introduced in the several famous papers for modeling and performance analysis of computer and communication systems are widespread since it allows an extremely efficient solution compared with any other analysis approach. Nowadays, a formal approach such as Petri Nets enable one to describe complex discrete event systems precisely and thus allows one to perform both qualitative and quantitative analysis, scheduling and discrete-event control of them. In fact, Petri Net is a very compact representation of Markov techniques and queues, in particular, however, the problem is usually the huge size of the state space.

The alternative approach is to incorporate the queues into the high-level specification, namely, NetLevel. However, the ordinary Petri Nets cannot represent queueing disciplines as in queueing networks. On the other hand, the ordinary Petri Nets allow for the use of synchronization and fork/join behavior both of that cannot be described in queueing networks. Combining Queueing Networks and Petri Nets have been studied at a few of papers. Falko and Peter [1] proposed queueing Petri Nets with product form solution. They introduced product form QPN which combine PSPN and PQN in one model formalism, namely the QPN formalism. Furthermore, they presented an arrival theorem and discussed exact aggregation in PQPNs. However, in that model formalism, the Petri Net and Queueing Network are loosely couple. Nevertheless, it also serves as a good tutorial paper. Erol Gelenbe et al[2, 3, 4] proposed a G-network including negative and positive customers. The G-network is a good original approach, but some important items have not been considered. Such as a new negative may be generated to indicate the positive has finished its service.

In this paper, we proposed a Hybrid Petri Net (HPN) that extends the framework of the original colored generalized stochastic Petri Net by hiding queues in special places. The Hybrid Petri Net contains ordinary tokens and control tokens. Both of ordinary tokens and control tokens can be exogenous, or can be obtained by a Markovian movement of a token from one place to another place after firing of transition. A control token forces an ordinary token to move instantaneously to another place according to a Markovian routing rule. We show that this new class of Petri Net has product form stationary solution, and establish the non-linear token flow equations that govern it. The stability of Hybrid Petri Net is discussed in this new context.

The organization of this paper is as follows. Section 2 describes the model of queueing service using Hybrid Petri Net. In Section 3 we will prove that this new class of Petri Nets have product form. Due to the non-linearity of the traffic equations for these models, the existence and uniqueness of their solution have to be addressed. In addition, a product form of nonpreemptive priority queueing also be discussed. Section 4 is concluding remark.

\section{The Model}

We proposed a Hybrid Petri Net (HPN) that extends the framework of the original colored generalized stochastic Petri Net by hiding queues in special places. The Hybrid Petri Net contains ordinary tokens and control tokens. Both of ordinary tokens and control tokens can be exogenous, or can be obtained by a Markovian movement of a token from one place to another place after firing of transition. A control token 


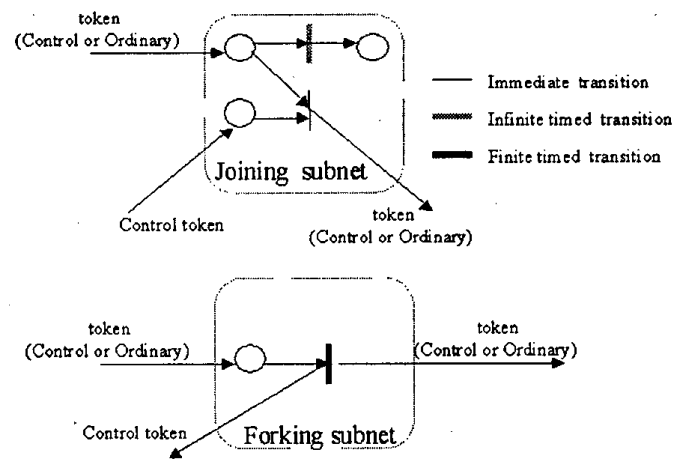

Figure 1: Forking subnet and joining subnet

forces an ordinary token to move instantaneously to another place according to a Markovian routing rule.

In our proposed Hybrid Petri Net, there are two special elementary types of subnet as shown in Fig. 1 in spit of ordinary places and transitions, namely, joining subnet and forking subnet. The service time of joining subnet is infinite. The service time of forking subnet follow some probability distribution. The service time of forking subnet is zero. When a token enter a joining subnet, the token will receive no service from the server and will stay in subnet for blocking. In our queueing services model, we allow work both to be moved from a subnet to another without service, and destroyed, under the effect of control token which are either exogenous, or generated by the ordinary tokens after service. Before presenting our queueing model the necessary terminology is introduced. Let,

$C=$ number of token classes.

$K=$ number of subnet (resources modeled as queueing centers or infinite server).

$S_{k c}=$ mean number of external visits made by a class $c$ ordinary token at subnet $k$.

$Z_{k c}=$ mean number of external visits made by a class $c$ control token at subnet $k$.

$N_{k c}=$ number of class $c \in(1, \ldots, C)$ tokens at subnet $k$.

$V_{k c}=$ mean number of visits made by a class ordinary token at subnet $k$.

$R_{k c}=$ mean service demand rate per visit for a class $c$ ordinary token at subnet $k$

As shown in Fig. 2, a token(control token or ordinary token) of class which leaves forking subnet $i$ (after finish service) goes to subnet $j$ as a token of class $l$ with probability $p(i, j)(k, l)$, it may depart from the network with probability $P(i)(k)=1-\sum_{j} \sum_{l} p(i, j)(k, l)$. A token (control token or ordinary token) of class $\mathrm{k}$ which

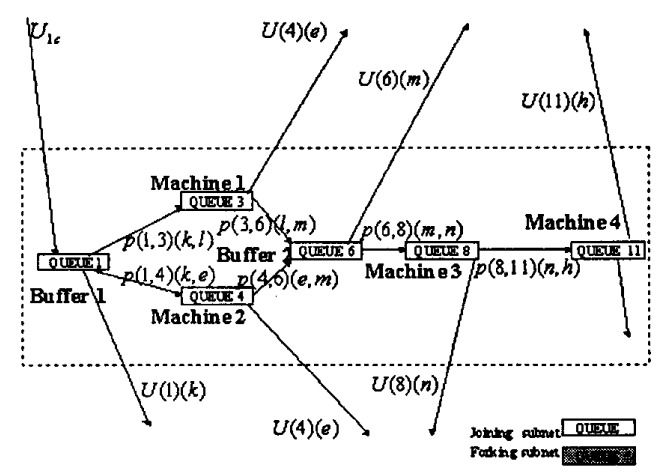

Figure 2: A queueing service model of Jackson Queueing Network

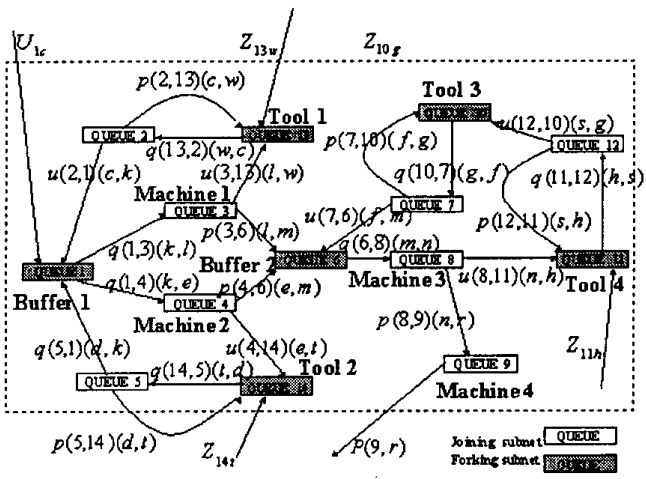

Figure 3: A queueing service model of a production network

leaves joining subnet $i$ (after finish service) goes to subnet $j$ as a token of class $l$ with probability zero.

The arriving control token triggers the instantaneous passage of a token(control token or ordinary token) of class $k$ from joining subnet $i$ to class $l$ of some other subnet $j$ with probability $q(i, j)(k, l)$. With probability $Q(i)(k)=1-\sum_{j} \sum_{l} q(i, j)(k, l)$, it forces a token to leave the network. After triggering the instantaneous passage of a token, the arriving control token is destroyed. The arriving control token triggers the instantaneous passage of a token(control token or ordinary token) of class $k$ from forking subnet $i$ to class $l$ of some other subnet $j$ with probability zero.

When a token(control token or ordinary token) of class $k$ which leaves forking subnet $i$ (after finish service), it generates a new control token from forking subnet $i$ to class $l$ of some other subnet $j$ with probability $u(i, j)(k, l)$. it may not generate a new control token into the network with probability $U(i)(k)=1-\sum_{j} \sum_{l} u(i, j)(k, l)$ When a token(control token or ordinary token) of class $k$ which leaves join- 


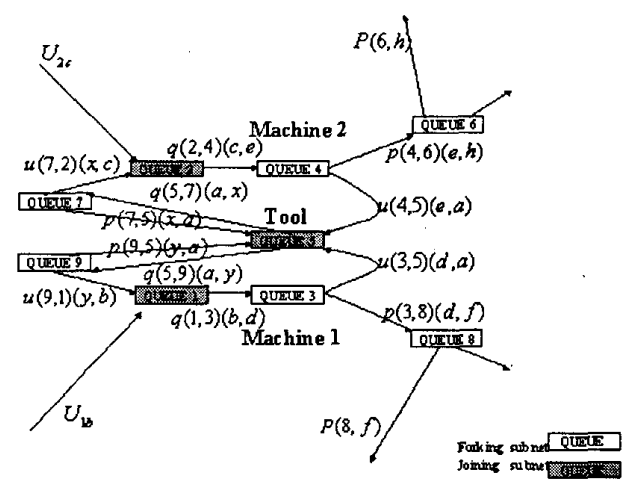

Figure 4: A queueing service model of share resource

ing subnet $i$ (after finish service), it generates a new control token from joining subnet $i$ to class $l$ of some other subnet $j$ with probability zero.

\section{Analysis Result}

The system as a whole is Markov because we assume the arrival processes are all Poisson and service processes are exponential, therefore, there can be following types of transitions.

1 No changes in system state.

2 An external visit made by a control(ordinary) customer at forking(joining) queue $k$.

3 An external visit made by a control customer at joining queue $k^{\prime}$, and the control customer triggers the instantaneous passage of a control(ordinary) customer from joining queue $k^{\prime}$ to forking(joining, outside) queue $k$.

4 Transfer of a control(ordinary) token (after service) from forking subnet $k^{*}$ to forking(joining, outside network) subnet $k$ ". A new control token generated from forking subnet $k^{*}$ outside the network, when the control(ordinary) token leaves forking subnet $k^{*}$ (after service) .

5 Transfer of a control(ordinary) token (after service) from forking subnet $k^{*}$ to forking(joining, outside network)subnet $k$ ". A new control token generated from forking subnet $k^{*}$ to joining subnet $k^{\prime}$, when a control(ordinary) token leaves forking subnet $k^{*}$ (after service). The new control token triggers the instantaneous passage of a control(ordinary) token from joining subnet $k^{\prime}$ to forking(joining, outside network) subnet $k$.

For a stable queueing system, the rate of tokens entering the system should equal to the rate of tokens leaving the system if we observe it for a sufficiently long period. Therefore, we can write down the traffic equations for each node of subnet as well as the network as whole. For each node of forking subnet, we have

$\forall k \in\left\{K^{\prime}, \ldots, K\right\}$

$$
\begin{aligned}
\alpha_{k c} & =S_{k c}+Z_{k c}+\sum_{k^{\prime}=1}^{K^{\prime}} \sum_{c^{\prime}=1}^{C} \beta_{k^{\prime} c^{\prime}} q\left(k^{\prime}, k\right)\left(c^{\prime}, c\right) \\
& +\sum_{k^{\prime}=K^{\prime}}^{K} \sum_{c^{\prime}=1}^{C} \frac{1}{2} \alpha_{k^{\prime} c^{\prime}} q\left(k^{\prime}, k\right)\left(c^{\prime}, c\right)
\end{aligned}
$$

On the other hand, for each node of joining subnet, we have $\forall k \in\left\{1, \ldots, K^{\prime}\right\}$

$$
\begin{aligned}
\beta_{k c} & =S_{k c}+Z_{k c}+\sum_{k^{\prime}=K^{\prime}}^{K} \sum_{c^{\prime}=1}^{C} \frac{1}{2} \alpha_{k^{\prime} c^{\prime}} p\left(k^{\prime}, k\right)\left(c^{\prime}, c\right) \\
& +\sum_{k^{\prime}=K^{\prime}}^{K} \sum_{c^{\prime}=1}^{C} \frac{1}{2} \alpha_{k^{\prime} c^{\prime}} u\left(k^{\prime}, k\right)\left(c^{\prime}, c\right)
\end{aligned}
$$

Let $N_{k c}(t)$ be the number of number of class tokens of subnet $k$ at timet. The system state is denoted as $\left\{N_{11}(t), \ldots, N_{1 C}(t), \ldots, N_{K 1}(t), \ldots, N_{K C}(t) T \geq\right.$ $0\}$. We assume the arrival process is Poisson and the service time distributions are exponential, we can have only the following events happening in an incremental time interval $\Delta t$. It satisfies a system of Chapman-Kolmogorov equations, and its steady-state distribution $p[N]=p\left[N_{11}, \ldots, N_{K C}\right]=$ $\lim _{t \rightarrow 0} p\left[N_{11}(t)=N_{11}, \ldots, N_{K C}(t)=N_{K C}\right]$ To begin with, we define the following notations; $\tilde{N}=\left(N_{11}, \ldots, N_{1 C}, \ldots, N_{K 1}, \ldots, N_{K C}\right) \quad \tilde{1}_{k c}=$ $\left(0, \ldots, N_{k c}, \ldots, 0\right) \tilde{N}-\tilde{\mathfrak{I}}_{k c}=\left(N_{11}, \ldots, N_{1 C}, \ldots, N_{k c}-\right.$ $\left.1, \ldots, N_{K C}\right) \tilde{N}+\tilde{1}_{k c}=\left(N_{11}, \ldots, N_{1 C}, \ldots, N_{k c}+\right.$ $\left.1, \ldots, N_{K C}\right)$

we focus our attention on the change in system state $\tilde{N}$ in an incremental time interval $\Delta t$. Therefore,

$$
\begin{aligned}
& p[\tilde{N}(t+\Delta t)] \\
= & p[\tilde{N}(t)]\left(1-\sum_{k=1}^{K} \sum_{c=1}^{C} R_{k c} \Delta t-\sum_{k=1}^{K} \sum_{c=1}^{C} S_{k c} \Delta t\right. \\
& \left.-\sum_{k=1}^{K} \sum_{c=1}^{C} Z_{k c} \Delta t\right) \\
+ & \sum_{k=1}^{K} \sum_{c=1}^{C} p\left[\tilde{N}(t)+\tilde{1}_{k c}\right]\left(S_{k c} \Delta t+Z_{k c} \Delta t\right) \\
+ & \sum_{k^{\prime}=1}^{K} \sum_{c^{\prime}=1}^{C} \sum_{k=1}^{K+1} \sum_{c=1}^{C} p\left[\tilde{N}(t)+\tilde{1}_{k^{\prime} c^{\prime}}-\tilde{1}_{k c}\right] \\
& Z_{k^{\prime} c^{\prime}} q\left(k^{\prime}, k\right)\left(c^{\prime}, c\right) \Delta t \\
+ & \sum_{k^{*}=K^{\prime}}^{K} \sum_{c^{*}=1}^{C} \sum_{k^{\prime \prime}=1}^{K+1} \sum_{c^{\prime \prime}=1}^{C} p\left[\tilde{N}(t)+\tilde{1}_{k^{*} c^{*}}-\tilde{1}_{k c}\right] \\
& Z_{k^{\prime \prime} c^{\prime \prime}} R_{k^{*} c^{*}} p\left(k^{*}, k^{\prime \prime}\right)\left(c^{*}, c^{\prime \prime}\right) U\left(k^{*}\right)\left(c^{*}\right) \Delta t
\end{aligned}
$$




$$
\begin{aligned}
+ & \sum_{k^{*}=K^{\prime}}^{K} \sum_{c^{*}=1}^{C} \sum_{k^{\prime \prime}=1}^{K} \sum_{c^{\prime \prime}=1}^{C} \sum_{k^{\prime}=1}^{K^{\prime}} \sum_{c^{\prime}=1}^{C} \sum_{k=K^{\prime}}^{K} \sum_{c=1}^{C} \\
& p\left[\tilde{N}(t)+\tilde{1}_{k^{*} c^{*}}-\tilde{1}_{k^{\prime \prime} c^{\prime \prime}}+\tilde{1}_{k^{\prime} c^{\prime}}-\tilde{1}_{k c}\right] \\
& R_{k^{*} c^{*}} p\left(k^{*}, k^{\prime \prime}\right)\left(c^{*}, c^{\prime \prime}\right) u\left(k^{*}, k^{\prime}\right)\left(c^{*}, c^{\prime}\right) q\left(k^{*}, k^{\prime}\right)\left(c^{*}, c^{\prime}\right) \Delta(t)
\end{aligned}
$$

Rearranging terms in $\mathrm{Eq} 1$, dividing the equation by $\Delta t$, taking limits, dropping the time parameter and setting the derivative of the joint probability density function to zero; we obtain $\mathrm{Eq} 2$

$$
\begin{aligned}
& p[\tilde{N}(t)]\left(\sum_{k=1}^{K} \sum_{c=1}^{C} R_{k c}+\sum_{k=1}^{K} \sum_{c=1}^{C} S_{k c}+\sum_{k=1}^{K} \sum_{c=1}^{C} Z_{k c}\right) \\
= & \sum_{k=1}^{K} \sum_{c=1}^{C} p\left[\tilde{N}(t)+\tilde{1}_{k c}\right]\left(S_{k c}+Z_{k c}\right) \\
+ & \sum_{k^{\prime}=1}^{K} \sum_{c^{\prime}=1}^{C} \sum_{k=1}^{K+1} \sum_{c=1}^{C} p\left[\tilde{N}(t)+\tilde{1}_{k^{\prime} c^{\prime}}-\tilde{1}_{k c}\right] \\
& Z_{k^{\prime} c^{\prime}} q\left(k^{\prime}, k\right)\left(c^{\prime}, c\right) \\
+ & \sum_{k^{*}=K^{\prime}}^{K} \sum_{c^{*}=1}^{C} \sum_{k^{\prime \prime}=1}^{K+1} \sum_{c^{\prime \prime}=1}^{C} p\left[\tilde{N}(t)+\tilde{1}_{k^{*} c^{*}}-\tilde{1}_{k c}\right] \\
& Z_{k^{\prime \prime} c^{\prime \prime}} R_{k^{*} c^{*}} p\left(k^{*}, k^{\prime \prime}\right)\left(c^{*}, c^{\prime \prime}\right) U\left(k^{*}\right)\left(c^{*}\right) \\
+ & \sum_{k^{*}=K^{\prime}}^{K} \sum_{c^{*}=1}^{C} \sum_{k^{\prime \prime}=1}^{K} \sum_{c^{\prime \prime}=1}^{C} \sum_{k^{\prime}=1}^{K^{\prime}} \sum_{c^{\prime}=1}^{C} \sum_{k=K^{\prime}}^{K} \sum_{c=1}^{C} \\
& p\left[\tilde{N}(t)+\tilde{1}_{k^{*} c^{*}}-\tilde{1}_{k^{\prime \prime} c^{\prime \prime}}+\tilde{1}_{k^{\prime} c^{\prime}}-\tilde{1}_{k c}\right] \\
& R_{k^{*} c^{*}} p\left(k^{*}, k^{\prime \prime}\right)\left(c^{*}, c^{\prime \prime}\right) u\left(k^{*}, k^{\prime}\right)\left(c^{*}, c^{\prime}\right) q\left(k^{*}, k^{\prime}\right)\left(c^{*}, c^{\prime}\lfloor 2)\right.
\end{aligned}
$$

\section{Product Form}

Consider the following of non-linear equations Eq 3 and Eq $4: \forall k \in\left\{K^{\prime}, \ldots, K\right\}$,

$$
\begin{aligned}
& \Lambda_{k c}=S_{k c}+Z_{k c} \\
+ & \sum_{k^{\prime}=1}^{K^{\prime}} \sum_{c^{\prime}=1}^{C} Z_{k^{\prime} c^{\prime}} \Psi_{k^{\prime} c^{\prime}} q\left(k^{\prime}, k\right)\left(c^{\prime}, c\right) \\
+ & \sum_{k^{\prime}=K^{\prime}}^{K} \sum_{c^{\prime}=1}^{C} R_{k^{\prime} c^{\prime}} \Psi_{k^{\prime} c^{\prime}} p\left(k^{\prime}, k\right)\left(c^{\prime}, c\right) U\left(k^{\prime}\right)\left(c^{\prime}\right) \\
+ & \left.\sum_{k^{*}=K^{\prime}}^{K} \sum_{c^{*}=1}^{C} \sum_{k^{\prime \prime}=1}^{K} \sum_{c^{\prime \prime}=1}^{C} \sum_{k^{\prime}=1}^{K^{\prime}} \sum_{c^{\prime}=1}^{C} R_{k^{*} c^{*}} \Psi_{k^{\prime} c^{\prime}}\right)\left(c^{*}, c^{\prime \prime}\right) u\left(k^{*}, k^{\prime}\right)\left(c^{*}, c^{\prime}\right) q\left(k^{*}, k^{\prime}\right)\left(c^{*}, c^{\prime}\right) \\
\Omega_{k c}=R_{k c} & \\
+ & \sum_{k^{*}=1}^{K} \sum_{c^{*}=1}^{C} \sum_{k^{\prime}=1}^{K^{\prime}} \sum_{c^{n}=1}^{C} R_{k c} \Psi_{k c} p\left(k, k^{*}\right)\left(c, c^{*}\right) u\left(k, k^{\prime}\right)\left(c, c^{\prime}(3)\right.
\end{aligned}
$$

On the other hand, for each node of joining subnet, we have $\forall k \in\left\{1, \ldots, K^{\prime}\right\}$,

$$
\begin{aligned}
& \Lambda_{k c}=S_{k c}+Z_{k c} \\
&+ \sum_{k^{\prime}=K^{\prime}}^{K} \sum_{c^{\prime}=1}^{C} R_{k^{\prime} c^{\prime}} \Psi_{k^{\prime} c^{\prime}} p\left(k^{\prime}, k\right)\left(c^{\prime}, c\right) U\left(k^{\prime}\right)\left(c^{\prime}\right) \\
&+ \sum_{k^{*}=K^{\prime}}^{K} \sum_{c^{*}=1}^{C} \sum_{k^{\prime \prime}=1}^{K} \sum_{c^{\prime \prime}=1}^{C} \sum_{k^{\prime}=1}^{K^{\prime}} \sum_{c^{\prime}=1}^{C} R_{k^{*} c^{*}} \Psi_{k^{\prime} c^{\prime}} \\
& p\left(k^{*}, k^{\prime \prime}\right)\left(c^{*}, c^{\prime \prime}\right) u\left(k^{*}, k\right)\left(c^{*}, c\right) q\left(k, k^{\prime}\right)\left(c, c^{\prime}\right) \\
& \Omega_{k c}=\sum_{k^{\prime}=K^{\prime}}^{K} \sum_{c^{\prime}=1}^{C} Z_{k c} \Psi_{k c} q\left(k, k^{\prime}\right)\left(c, c^{\prime}\right) \\
&+\quad \sum_{k^{*}=K^{\prime} c^{*}=1}^{K} \sum_{k^{\prime \prime}=1}^{C} \sum_{c^{\prime \prime}=1}^{K} \sum_{k^{\prime}=1}^{K} \sum_{c^{\prime}=1}^{C} R_{k^{*} c^{*}} \Psi_{k^{\prime} c^{\prime}} \\
& p\left(k^{*}, k^{\prime \prime}\right)\left(c^{*}, c^{\prime \prime}\right) u\left(k^{*}, k\right)\left(c^{*}, c\right) q\left(k, k^{\prime}\right)\left(c, c^{\prime}\right)
\end{aligned}
$$

Theorem 1 If a non-negative solution $\Lambda_{k c}$ and $\Omega_{k c}$ exist such that $\Lambda_{k c}<\Omega_{k c}$ for $\forall c \in\{1, \ldots, C\}$, then

$$
p[\tilde{N}]=G \prod_{k=1}^{K} \prod_{c=1}^{C}\left(\frac{\Lambda_{k c}}{\Omega_{k c}}\right)^{N_{k c}}
$$

Proof : we omit the proof, which follows similar technique $[2,3,4,8,7]$.

\section{Stability}

Unlike Jackson networks, the token flow equations of the model we consider are nonlinear. Therefore, issues of existence and uniqueness of the solutions have to be examined. In particular, our essential result depends on the existence of solution(Eq 34 ). Thus, the existence and uniqueness of solutions to these traffic equations is central to our work. Note that if existence is established, then uniqueness follows easily for a simple reason. We are dealing with the stationary solution of a system of Chapman-Kolmogorov equations, which is known to be unique, if it exists.

Theorem 2 The solution $\left\{\Lambda_{k c}, \Omega_{k c}\right\}$ for $\forall c \in$ $\{1, \ldots, C\}, \forall k \in\{1, \ldots, K\}$, always exists.

Proof : we omit the proof, which follows similar technique $[2,3,4,8,7]$.

Theorem 2 provides grounds for computing the arrival rates of tokens and signals to each subnet, since it guarantees that these can always be found by solving the nonlinear equation. This issue is only a basis for determining whether the network solution in steadystate can be obtained. Indeed. it is easy to imagine situations where once the arrival rate are computed, on discovers that the network is unstable.

\section{Non-preemptive Priority Queueing}

So far, we only identifies the token classes of our Hybrid Petri Net. However in real-life, we often encounter sit- 
uations where certain tokens are given a greater privilege. They receive their services before others. The arriving tokens belong to one of different priority classes. We adopt the convention that the larger the value of the index associated with the priority group, the higher is the so-called priority associated with that group. Then, we associate with a token from priority group a numerically valued priority function at the time instant. The choice is made in favor of that token with largest valued priority function. Thus, all ties are broken on an FCFS basis.

A token of class $c$ who arrives at the subnet $k$, let us consider the waiting time of the arriving token as the following :

1 When a class $c$ token arrives, the probability that the token finds a class $c^{\prime}$ token in services is $\rho_{k c^{\prime}}=$ $\Lambda_{k c^{\prime}} R_{k c^{\prime}}$, therefore the mean residual service time $\gamma$ is given by weighted sum of the residual service time of each class. $\gamma=\frac{1}{2} \sum_{c^{\prime}=1}^{C} \Lambda_{k c^{\prime}} R_{k c^{\prime}}{ }^{2}$

2 The mean total service time of tokens in the same class $c$ before the arriving token. That is $N_{k c} R_{k c}$.

3 The mean total service time of tokens in the higher priority class $c^{\prime}$ before the arriving token. That is $\sum_{c^{\prime}=c+1}^{N_{k c^{\prime}}} R_{k c^{\prime}}$.

4 The mean total service time of tokens in the higher priority class $c^{\prime}$ arriving during the token is waiting in queue. That is $\sum_{c^{\prime}=c+1}^{\Lambda_{k c^{\prime}}} W_{k c^{\prime}} R_{k c^{\prime}}$. Then, we have

$$
W_{k c}=\frac{\gamma}{\left(1-\sum_{c^{\prime}=c+1}^{C} \rho_{k c^{\prime}}\right)\left(1-\sum_{c^{\prime}=c}^{C} \rho_{k c^{\prime}}\right)}
$$

, In addition, we can get the following.

Theorem 3 The product form of non-preemptive priority queueing can be described as

$$
p[\tilde{N}]=G \prod_{k=1}^{K} \prod_{c=1}^{C}\left(\frac{\Lambda_{k c}}{\Omega_{k c}+W_{k c}}\right)^{\Lambda_{k c} W_{k c}}
$$

Proof : we omit the proof.

\section{Concluding Remark}

In this paper, we describes the model of queueing service using Hybrid Petri Net. We will prove that this new class of Petri Nets have product form. Due to the non-linearity of the traffic equations for these models, the existence and uniqueness of their solution have also to be addressed. In addition, a product form of non-preemptive priority queueing also be discussed.

So far we consider a stable queueing system in our hybrid Petri Net model. However in real-applications, it is encountered that situations where the input parameter of system model changed very frequently. The balance equations may not exist and steady-state behavior may be violated. We review the Markov chain $X_{k}$ that is irreducible, aperiodic and timehomogeneous is said to be ergodic. For an ergodic Markov chain, the limiting probabilities always exist and are independent of the initial state probability.

$$
\pi_{i}=\lim _{k \rightarrow \infty} \pi_{i}^{(k)}=\lim _{k \rightarrow \infty} P\left[X_{k}=i\right]
$$

.When we estimating not long run or not steady state, measures of performance. The following should be considered.

1 For fixed time interval, different random number will lead to different performance. The random number depends on the time interval and initial conditions, such as the initial marking status of Petri Nets.

2 For the random number, we can have the assumption that the random number can be estimated as a general statistical distribution plus some bias. As time is increased, the estimator becomes more precise. The bias will be reduced.

Finally, there are many future work. One is development of throughput approximation technique based on interpolation or MVA [5]. The others are to study the queueing under the environment where steady-state behavior is violated.

\section{REFERENCES}

[1] Falko Bause and Peter Buchholz, "Queueing Petri Nets with Product Form Solution", Performance Evaluation, Vol. 32, pp. 265-299, 1998.

[2] Erol Gelenbe, "Product-Form Queueing Networks with Negative and Positive Customers", J. Appl. Prob. , Vol. 28, pp. 656-663, 1991.

[3] Erol Gelenbe, "G-Networks with Triggered Customer Movement", J. Appl. Prob. , Vol. 30, pp. 742-748, 1993.

[4] Jean-Michel Fourneau, Erol Gelenbe and Rina Suros, "G-Networks with Multiple Classes of Negative and Positive Customers", Theoretical Computer Science, Vol. 155, pp. 141-156, 1996.

[5] Matteo Sereno, "Approximate Mean Value Analysis for Stochastic Marked Graphs.", IEEE Tran. on Software Eng., Vol. 22, No. 9, Sep. 1996, pl). 654-664.

[6] Alan A. Desrochers and Robert Y. AI-Jaar, "Application of Petri Nets in Manufacturing Systems: Modeling, Control, and Performance Analysis.", IEEE Press. 
[7] Ng Chee Hock, "Queueing Modeling Fundamentals.", John Wiley 8 Sons Press.

[8] Leonard Kleinrock, "Queueing Systems Volume 1 : Theory.", John Wiley \& Sons Press.

[9] Leonard Kleinrock, "Queueing Systems Volume 2 : Computer Applications .", John Wiley \& Sons Press. 